\title{
Music and People with Tendencies to Depression
}

SANDRA GarRido

University of Melbourne, Melbourne, Australia

Emery Schubert

University of New South Wales, Sydney, Australia

DEPRESSION IS OFTEN ASSOCIATED WITH A REDUCED motivation to engage in behavior that will improve one's mood. This paper presents a study in which 175 university students listened to a self-selected piece of music on Youtube that made them sad. Post- and prelistening scores of depressed mood on the Profile of Mood States (POMS) were taken, as were measures of rumination and scores on the Like Sad Music Scale (LSMS). Results indicate that listening to sad music via this medium can significantly increase feelings of depression in people with a tendency to depression (as suggested by high rumination scores). Furthermore, people with a tendency to depression demonstrate a liking for such music despite the potentially unhealthy consequences of listening to it.

Received: November 10, 2013, accepted March 15, 2014.

Key words: depression, mood regulation, rumination, absorption, sad music

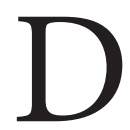

EPRESSION IS OFTEN DESCRIBED AS A disorder of affect dysregulation, involving the use of less effective strategies for regulating affective responses and a reduced motivation to engage in activities that could improve one's mood (Forbes \& Dahl, 2005). People who are depressed may have a higher threshold for the activation of positive affect, and experience positive affect in less intensity and for shorter durations than healthier people. They may also have a low activation threshold for negative affect and experience it more intensely and for longer periods.

One of the primary behaviors associated with depression is rumination (Nolen-Hoeksema, 1991). In fact, the presence of rumination is one of the strongest predictors of clinical depression (Calmes \& Roberts, 2007; Nolen-Hoeksema \& Morrow, 1993). Rumination, in the sense it is used in this paper, is an involuntary focus on negative and pessimistic thoughts (Conway, Csank,
Holm, \& Blake, 2000; Joorman, 2005). People with a propensity to ruminate and people with depression tend to have a bias towards stimuli that includes content of a negative valence (Gotlib, Krasnoperova, Neubauer Yue, \& Joormann, 2004). For example, people with depression form more negative sentences from scrambled words than non-depressed participants even when they are not in a negative mood (Wenzlaff \& Bates, 1998), tend to interpret facial expressions as conveying negative emotions (Raes, Hermans, \& Williams, 2006), and demonstrate stronger recall for negatively biased memories (Lyubomirsky, Caldwell, \& Nolen-Hoeksema, 1998). The evidence suggests that engaging in rumination can extend the length of depressive episodes (NolenHoeksema, 1991).

Studies indicate that this negative attentional bias, and the use of ineffective strategies for affect regulation, may also apply to music listening choices. Punkanen, Eerola, and Erkkila (2011), for example, found that depressed patients tended to perceive music as more negatively valenced than healthy participants. Bodner et al., (2007) found that depressed subjects showed a heightened response to sad music excerpts compared to a control group. Chen, Zhou, and Bryant (2007) found that ruminators in their laboratory experiment spent more time listening to distressing music than nonruminators. Questioning people about music use in real life settings, Garrido and Schubert (2013a) similarly found that rumination was correlated with spending a high proportion of music listening time on sad music, indicating that music listening may be used as a form of rumination.

The argument has been made that listening to music of negative valence can be a useful and healthy way to deal with negative emotions being experienced in real life. Van den Tol and Edwards (2013), for example, reported that mood enhancement is one of the key goals of listening to sad music described by their participants. Similar findings are reported by Saarikallio \& Erkkila (2007). Wilhelm and colleagues (2013) also reported that music was used by some of their depressed participants to dissipate their negative mood. Much of this literature, however, relies on reports of music listening goals or self-reported effects. Researchers seldom seem to make a distinction between goals for music listening and actual mood-related outcomes. The question remains as

Music Perception, volume 32, issue 4, pp. 313-321, issn 0730-7829, electronic issn 1533-8312. C 2015 by the Regents of the university of California All

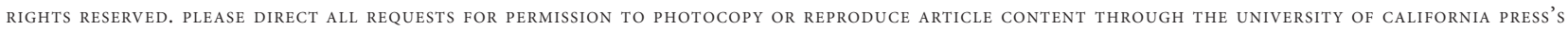
RIGHTS AND PERMISSIONS WEBSITE, HTTP://WWW.UCPRESSJOURNALS.COM/REPRINTINFO.ASP. DOI: 10.1525/MP.2015.32.4.313 
to whether the intended outcomes are actually achieved in all cases.

Some of the evidence seems to suggest that positive outcomes are not always achieved. In the same study in which Wilhelm et al. (2013) reported a dissipation of negative mood, they also reported that others experienced a worsening of negative mood. Further evidence indicates that musical engagement can also be a part of unhealthy patterns for dealing with negative emotions. Miranda and Claes (2009), for example, found that adolescents with depression may use music as a part of maladaptive coping strategies, such as emotion or avoidance coping styles. Garrido and Schubert (2013b) also found that listening to music evoking negative emotions was associated with an increase in depressed mood.

There are two possible reasons why some participants may report benefiting from engaging with music evoking negative emotions despite this evidence of its unhealthy effect. First, it may be that some people do obtain certain psychological benefits such as catharsis, the opportunity to reframe events in their mind, or to express their negative affective state when listening to music evoking negative emotions. Second, people with tendencies towards depression may be unaware of the effect the music has upon them and therefore report positive benefits. People who engage in ruminative behavior in other contexts demonstrate a belief that such behavior will result in being better able to understand their emotions or to avoid making similar mistakes in the future, despite evidence that the behavior does not result in an improved mood (Barnhofer, Kuehn, de JongMeyer, \& Williams, 2006). Therefore, it is possible that when music and music videos are used in a ruminative way, people may not always be aware of the negative effect it is having and may therefore tend to offer rational explanations for their maladaptive behavior.

Trapnell and Campbell (1999) outlined an important distinction between rumination and reflection, both of which involve focusing on thoughts that may be of a negative content. The distinction appears to lie largely in the degree to which people are able to work through and then detach from the negative thoughts. Ruminators, as discussed above, seem to find it difficult to disengage from negative thoughts once they arise, and negative emotions are perpetuated rather than being resolved. Reflection, on the other hand, involves a healthy process of thinking through negative emotions and thoughts and reaching some resolution, perhaps through strategies such as reappraisal. The individual is then able to progress towards a more positive mood rather than being caught up in a self-perpetuating cycle of negative thinking. This crucial distinction may at least in part, explain how it is that some people report benefiting from listening to music evoking negative emotions, while others do not.

The distinction between rumination and reflectiveness was proposed by Trapnell and Campbell as a solution to the self-absorption paradox: the fact that a tendency to be absorbed in thoughts about one's own emotions and experiences is often found to be a healthy, adaptive trait, but is also associated with neuroticism, depression, and poor self-esteem. It may also present a solution to the paradox found in much of the literature summarized above on negative emotions in music, namely, that some studies suggest that musical engagement can be part of unhealthy patterns of behavior, while other studies argue that positive benefits are to be gained by engaging with music evoking negative emotions.

"Absorption" is itself a trait that is present in individuals to a greater or lesser degree. As a trait, it can be described as the capacity to become immersed in activities or experiences with a narrowing of concentration in which one becomes less conscious of external surroundings and the passage of time (Eisen \& Lynn, 2001). It is not hard to imagine that the presence of strong levels of trait absorption could exaggerate the effects of rumination, making a person more likely to experience negative affect to an intense degree and less likely to be able to disengage from negative thoughts and stimuli. Since absorption is a prima facie enjoyable experience akin to "flow" (Privette, 1983; Rodriguez-Sanchez, Schauflei, Salanova, Cifre, \& Sonnenschein, 2011), it could even be that ruminators with strong capacities to absorption may find some kind of immediate pleasure in experiencing negative emotions more intensely despite its potentially detrimental effects on their mood.

\section{Aims}

This study aimed to further investigate the effect of listening to sad music on people with tendencies toward depression as indicated by high scores in rumination. We wanted to test whether people with a propensity to depression are aware of the effect that listening to sad music has upon their mood, and whether they argue that such behavior benefits them. We also wanted to examine whether absorption might exacerbate the effect of rumination. Specifically, we tested three hypotheses:

$\mathrm{H}^{1}$ : People with high scores in rumination will predict that listening to sad music will benefit them in some way.

$\mathrm{H}^{2}$ : People with high scores in rumination will not experience an improved mood after listening to sad music. 
$\mathrm{H}^{3}$ : People with high scores in rumination will demonstrate an attraction to sad music despite the fact that listening to it does not result in an improved mood.

\section{Method}

\section{PARTICIPANTS}

After discarding ineligible completions from an initial pool of 191 (derived from a University in Australia who participated in the study in exchange for course credit), 175 responses were collected for further analysis. Participants included 72 males and 103 females with a mean age of 20.9 years (range 18-48). Most had some experience playing a musical instrument or singing $(90.9 \%)$ with an average of 7.61 years of musical experience.

\section{PROCEDURE}

Participants were given a period of two weeks in which to complete an online survey that took approximately 20-30 min to complete. After describing the purpose of the study and citing the ethics approval number given to this project, listeners were requested to find URLs (such as for a YouTube video) for a piece of music that made them feel sad and one that made them happy. They were instructed not to listen to the music until specified points in the survey. Participants were also requested to attempt to complete the survey on a computer with a good sound system or with good quality headphones and in a private place. They were further instructed that the survey was to be completed in a single sitting. The survey software recorded the date and time that the survey was commenced and completed, and sixteen participants were omitted from analysis since these data indicated that they had likely not complied with the request to complete the survey in a single sitting (leaving a total of 175 participants).

Music videos on YouTube may sometimes be professionally produced film clips in which the singer is depicted miming the words to the song in which a story is acted out, or, they may be amateur productions in which the lyrics are scrolled across the screen or various animations are shown. However, defining the parameters of the musical stimuli that evoke negative emotions was beyond the scope of the current paper. Rather, in this study we were interested in examining the effect upon mood of engaging with music that could be described as "sad." Therefore, the subsequent questions focused on music listening, although it is possible that the extra musical factors inherent in the medium may also have contributed to the effects reported herein.

A set of questions relating to the predicted effect of listening to sad music were then asked, along with several personality, arousal and mood measures described below. Participants were then requested to listen to the sad music they had selected using the video URL they had provided. An automatic time stamp was generated by the survey software to allow examination of the time spent on this task as a check of compliance. All included participants spent an appropriate amount of time on this task (commensurate with the length of the self-selected pieces, based on the URL links they provided to the pieces), and therefore no further participants were eliminated from the study. A post-listening measure of mood was taken after listening to the self-selected sad music. Participants were then requested to listen to a piece of happy music so as to end the experiment in a positive condition. Demographic information was collected at the end of the survey.

\section{MEASURES}

The first block of questions was designed to examine whether people would predict negative or positive effects of listening to sad music on their mood (Predicted Effect of Sad Music, PESM). The question read, "What effect does listening to sad music have on you?" and participants were then instructed to rate ten statements according to their level of agreement on a 7-point scale from -3 (strongly disagree) to 3 (strongly agree) (see Table 1). The items were drawn from the Like Sad Music Scale (LSMS) designed by Garrido and Schubert (2013a). The LSMS was created by the authors to measure how much people like sad music as well as to identify specific motives for listening to it. The scale consists of 10 items that were rated on a 5-point scale from 1 (strongly disagree) to 5 (strongly agree). The authors reported high internal consistency (Cronbach's alpha $=.80$ ). The items for the PESM were adapted from the LSMS to investigate the participant's predictions of the effect of listening to sad music, rather than a liking for it in general. Items suggested either a predicted positive (items 1, 3, 5, 6, 9), negative (items $2,4,10$ ), or mood dependent effect (item 8).

Participants completed both the PESM and the LSMS. They then completed two questions relating to how much time they spent listening to music per week and how much time they spent listening to sad music per week (as per Garrido \& Schubert, 2013a).

Rumination and reflectiveness were measured using Trapnell and Campbell's Rumination Reflection Questionnaire (RRQ, 1999). Absorption was measured using a 12-item subscale from the Absorption, Intellectance, and Liberalism Questionnaire (AIT, Glisky \& Kihlstrom, 1993). Agreement with items on both scales was rated on 
TABLE 1. Survey Items Predicting the Effect of Listening to Sad Music

\begin{tabular}{|c|c|c|}
\hline Item No. & Item Text & Predicted Effect \\
\hline PESM01 & It makes me cry which makes me feel peaceful and relieved afterward. & Positive \\
\hline PESM02 & It reminds me of some sad things in my life which makes me feel sadder than I did before. & Negative \\
\hline PESM03 & $\begin{array}{l}\text { It gives me an opportunity to reflect on some sad things in my own life and then having } \\
\text { worked through those feelings, I feel better afterward. }\end{array}$ & Positive \\
\hline PESM04 & $\begin{array}{l}\text { I can relate to the person singing/playing the music. Its as if they were telling me their own } \\
\text { story. Its good to know that other people sometimes feel like me. }\end{array}$ & Positive \\
\hline PESM05 & I feel sad, but I enjoy just becoming immersed in the emotions of the moment. & Positive \\
\hline PESM06 & I feel sad, but somehow more alive. & Positive \\
\hline PESM07 & It makes me feel sad, and therefore I don't like listening to sad music. & Negative \\
\hline PESM08 & $\begin{array}{l}\text { If I am in a good mood I just enjoy listening to the music even if it is sad, } \\
\text { but if I am in a bad mood, sad music makes me feel even worse. }\end{array}$ & Mood Dependent \\
\hline PESM09 & $\begin{array}{l}\text { The sadness of the music helps me to release any negative emotions I am experiencing and } \\
\text { then I feel better. }\end{array}$ & Positive \\
\hline PESM10 & It makes me think of past events in my life, a bittersweet nostalgic experience. & Negative \\
\hline
\end{tabular}

a 5-point scale from 1 (strongly disagree) to 5 (strongly agree).

The Profile of Mood States (POMS, McNair, Lorr, \& Droppleman, 1971), which includes six subscales (Tension, Anger, Fatigue, Depression, Vigour and Confusion), was used as a measure of baseline mood state. The six subscales also generate a total "Mood Disturbance Index." Participants rated a list of adjectives according to how they felt "right now" on a scale of 0 (not at all) to 4 (extremely). Baseline measures were also taken on four dimensions relating to arousal and valence (Calm/Anxious, Tired/Energetic, Sleepy/Alert, Negative/Positive), based on multidimensional models of emotion (Eerola \& Vuoskoski, 2011; Thayer, 1978). These same measures were then used after listening to the self-selected sad music.

Scoring. Scores for each of the variables were first calculated. The scores for Rumination, Reflectiveness, and Absorption were calculated as described by the authors of those subscales. The median score for Rumination (3.53) was used as a splitting point for participants. We therefore created post hoc groups of high Ruminators (participants with scores of 3.53 and above, $n=88$ ) and low Ruminators (participants with scores $<3.53$, $n=86$ ). These groups would constitute a betweensubjects independent variable.

POMS scores were obtained for each of the two time points by first calculating each subscale, including the Depression subscale as described by McNair et al. (1971). While a total Mood Disturbance Index (MDI) can be obtained by adding all subscale scores (excluding Vigor) and then subtracting Vigor scores (a positive mood state), this part of the scoring was not necessary for our analyses since we were particularly interested in people with a tendency to depression.

A Percentage of Sad Music Listening Time (PSMLT) variable was also created by calculating the percentage of total listening time that participants spent listening to sad music.

\section{Results}

\section{PREDICTION OF THE EFFECT OF SAD MUSIC ON MOOD}

A MANOVA was conducted with Rumination Group (high and low) as the fixed factor and the 10 PESM items (see Table 1) as the dependent variables. Box's $\mathrm{M}$ test for homogeneity of variance was not significant, $F(55,954000)=1.299, p=.007$, and overall comparisons were significant, $F(10,163)=4.54, p<.001$, Wilks' lambda $=.78$.

Post hoc ANOVA tests of between-subject effects (see Table 2) revealed significant differences between high and low ruminators for Item PESM01 ("It makes me cry which makes me feel peaceful and relieved afterward"), PESM02 ("It reminds me of sad times in my life which makes me feel sadder"), PESM04 ("I can relate to the person singing/playing the music. Its as if they were telling me their own story. Its good to know that other people sometimes feel like me."), PESM05 ("It makes me feel sad but I enjoy being immersed in the emotions"), PESM06 ("I feel sad, but somehow more alive."), PESM09 ("The sadness of the music helps me to release any negative emotions that I am experiencing and then I feel better."), and PESM10 ("It makes me think of past events in my life, a bittersweet nostalgic experience"), with high ruminators giving higher ratings to all items than low ruminators (see Table 2). 
TABLE 2. Summary of Post Hoc ANOVA for Predicted Effects of Listening to Sad Music Between Low and High Ruminators

\begin{tabular}{|c|c|c|c|c|c|c|}
\hline & \multicolumn{2}{|c|}{ Low Ruminators } & \multicolumn{2}{|c|}{ High Ruminators } & \multirow[b]{2}{*}{$F$} & \multirow[b]{2}{*}{$p$} \\
\hline & $M$ & $S D$ & $M$ & $S D$ & & \\
\hline PESM01 & 3.45 & 1.77 & 4.05 & 1.70 & 5.07 & .03 \\
\hline PESM02 & 3.84 & 1.41 & 5.01 & 1.30 & 32.77 & .000 \\
\hline PESM03 & 4.63 & 1.42 & 4.86 & 1.56 & 1.09 & .30 \\
\hline PESM04 & 4.64 & 1.44 & 5.24 & 1.59 & 6.78 & .01 \\
\hline PESM05 & 5.13 & 1.34 & 5.72 & 1.30 & 8.63 & .004 \\
\hline PESM06 & 4.37 & 1.37 & 4.98 & 1.52 & 0.41 & .52 \\
\hline PESM07 & 2.85 & 1.66 & 2.69 & 1.53 & 3.09 & .08 \\
\hline PESM08 & 4.29 & 1.50 & 4.75 & 1.91 & 3.99 & .05 \\
\hline PESM09 & 4.38 & 1.57 & 4.85 & 1.53 & 13.46 & .000 \\
\hline PESM10 & 4.67 & 1.40 & 5.44 & 1.36 & 7.57 & .01 \\
\hline
\end{tabular}

Note: PESM = Predicted Effect of Sad Music

$d f=172$

PESM02 and PESM05 were similarly correlated with Rumination in the correlation analysis, $r(173)=.41$, $p<.001 ; r(173)=.27, p<.001$, respectively. These results partially support the first hypothesis in that some ruminators report an 'enjoyment' of the emotions experienced or some benefit to mood from listening to it, while others do not.

\section{ACTUAL EFFECT OF SAD MUSIC ON MOOD}

A mixed between-within subjects ANOVA was first conducted to assess the overall impact of the sad music listening on Depressed mood as measured by the POMS subscale. Time (baseline, post-sad music scores on Depression) was the within-subject factor, and Rumination Group (high and low) was the betweensubject factor. There was a significant main effect of Time on Depression, $F(1,172)=29.78, p<.001$, Wilks' lambda $=.85$, partial eta squared $=.15$. There was also a significant interaction effect between Time and Rumination Group, indicating that the effect upon high ruminators of musical engagement differed from the effect upon low ruminators, $F(1,172)=5.66, p=$ .018 , Wilks' lambda $=.97$, partial eta squared $=.03$. The test of between-subject effects indicated that there was also a significant main effect of Rumination Group on Depression scores, $F(1,172)=20.77, p<.001$, partial eta squared $=.11$ (see Figure 1 and Table 3 ). These results support the second hypothesis that people with high scores in rumination would not experience an improved mood after listening to sad music.

\section{ATTRACTION TO SAD MUSIC}

To test our third hypothesis a standard multiple regression analysis was performed with LSMS scores as the dependent variable and Rumination, Reflectiveness,

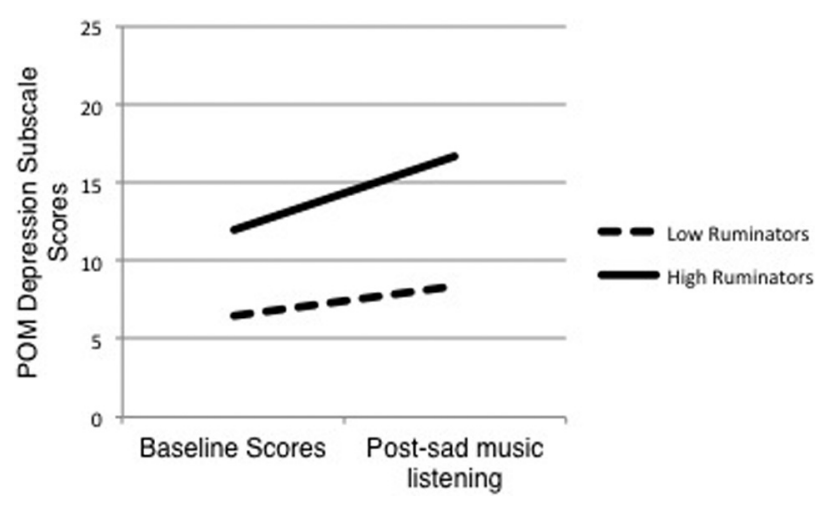

FIGURE 1. Low and high ruminator scores on POMS Depression Subscale at baseline and post-sad music.

TABLE 3. Rumination Group Scores on POMS Depression Subscale Across Three Time Points

\begin{tabular}{lrrrrr}
\hline & \multicolumn{2}{c}{ Baseline } & & \multicolumn{2}{c}{ Post-sad } \\
\cline { 2 - 3 } \cline { 6 - 6 } \cline { 5 - 6 } & \multicolumn{1}{c}{$M$} & \multicolumn{1}{c}{$S D$} & & \multicolumn{1}{c}{$M$} & \multicolumn{1}{c}{$S D$} \\
\hline Low Ruminators & 6.49 & 9.74 & & 8.34 & 8.85 \\
High Ruminators & 11.99 & 11.69 & & 16.70 & 12.39 \\
\hline
\end{tabular}

Absorption, and PSMLT as predictors. As shown in Table 3, PSMLT made the strongest contribution to LSMS (standardized beta $=.41, p<.001$ ). Absorption and Rumination made the next strongest, significant contributions (at $p<.05$ ) (with standardized beta's of .21 and .19 respectively). Reflectiveness was the weakest predictor retained in the model (standardized beta $=$ $0.16, p=.029)$. The model accounted for $35.6 \%$ of the variance (adjusted $R^{2}$ ). 
TABLE 4. Predictors for Standard Multiple Regression Model of the Like Sad Music Scale

\begin{tabular}{lrrrrr}
\hline Dependent & $B$ & $S E$ & Beta & T & $p$ \\
\hline (Constant) $_{\text {Rumination }}^{* *}$ & 14.11 & 0.53 & & 5.58 & 2.91 \\
Reflectiveness $_{\text {Absorption }^{* *}}$ & 1.63 & 0.56 & 0.19 & 2.20 & .001 \\
PSMLT $^{* *}$ & 1.32 & 0.60 & 0.16 & 2.95 & .03 \\
\hline
\end{tabular}

Notes: $B$ denotes unstandardized coefficients. Beta denotes standardized coefficients. $S E$ denotes standard error of Beta. ${ }^{* *}$ denotes variable significant at $p=.01$

Pearson's correlation analyses indicated a significant coefficient between Rumination score (as a continuous variable) and the LSMS, suggesting that high ruminators demonstrated a liking for sad music, $r(173)=.31$, $p<.001$. However, given that the LSMS was also correlated with Absorption, $r(173)=.37, p<.001$, and that a liking for sad music is not necessarily maladaptive (Garrido \& Schubert, 2013a), we decided to test for partial correlations. When a partial correlation controlling for Rumination was conducted with the LSMS and Absorption, $r$ fell slightly to .33 . However, when the partial correlation was conducted with the LSMS and Rumination controlling for Absorption, $r$ dropped to .25 , indicating that the relationship between the LSMS and Rumination is mediated by Absorption. In terms of the regression analysis this further supports the interpretation that the ruminators have a tendency to become absorbed in self-selected sad music, potentially drawing them into behavior that does not necessarily improve mood.

Since Absorption was once again correlated to the same items; PSM02: $r(173)=.29, p<.001$; PSM05: $r(173)=.33, p<.001$, it was decided to conduct partial correlations on these items too. We thus hoped to investigate the relationship between absorption and rumination on the predicted effect of listening to sad music. When controlling for Rumination, the coefficient for both items dropped but remained significant; (PSM02: $r(173)=.23, p=.002$; PSM05: $r(173)=.29, p<001$. However when Absorption was partialed out, the correlation coefficients between the items and Rumination also dropped but remained significant; PSM02: $r(173)=$ $.37, p<.001$; PSM05: $r(173)=.22, p=.003$. Overall, the strongest relationships were retained between PSM02 and Rumination and PSM05 and Absorption.

\section{Discussion}

We hypothesized that people with high scores in Rumination would predict some kind of benefit from engagement with sad music. The results partially supported this hypothesis in that people with high scores in rumination rated items predicting psychological benefit significantly higher than low ruminators. However, they also rated one item predicting feeling sadder higher than low ruminators.

We further hypothesized that people with high scores in rumination would not experience any improvement in mood after listening to sad music. The hypothesis was supported by the data, with high ruminators experiencing significant increases in depressed mood as measured by the POMS after listening to their selfselected YouTube video. This replicates the findings of Garrido and Schubert (2013b).

While no clinical assessment of depression was involved in this study, it is quite likely that participants on the high end of the spectrum for both Rumination and for the POMS Depression subscale are people with depression at clinical levels. In an unpublished study, Trapnell (2010) reported that participants with mild depression levels as measured by the Beck Depression Inventory (Beck, Ward, Mendelsohn, Mock, \& Erbaugh, 1961) had a mean score of 3.79 on the Rumination subscale of the RRQ. It is therefore likely that our high Rumination group, which had a score of 3.53 or above, could be categorized as having mild to severe depression, as did the sample used by Trapnell (2010). Studies also indicate that scores above 7 on the POMS Depression subscale can be equivalent to clinical levels of depression (Wilkins et al., 1995). As illustrated by Table 2, it can be seen that the High Rumination group stayed above this level both before and after musical engagement.

While this study did not examine the long-term effect of sad music, the results do suggest that people with a predisposition towards rumination and depression do not benefit at least in terms of immediate mood improvement from listening to sad music. This is particularly interesting in light of the fact that negative affect may tend to be more enduring, and that positive affect is generally less easily triggered in people with depression.

The results also suggest that some ruminators are aware of the fact that sad music makes them feel worse 
while others infer some benefits from listening to sad music. It is possible that while the immediate impact of listening to sad music as measured by the POMS may be negative, the long-term benefits may be more beneficial, which may be what participants were predicting. However, there are some other possible explanations for the discrepancies between predicted and actual effect.

First, people differ as to their levels of cognitive insight, meaning that individuals have different degrees of awareness of their own mental condition (Beck, Baruch, \& Balter, 2004; Riggs, Grant, Perivoloitis, \& Beck, 2012). Consequently, some people may be more aware than others of the negative impact listening to sad music can have on their moods. Second, it is possible that some people may actually enjoy a heightened feeling of sadness despite the fact that it does not have a positive effect on their moods. The fact that some ruminators claim that immersion in negative emotions is enjoyable, is a fascinating finding. It is logical that engaging in behavior, even that which is maladaptive, must be reinforced in the individual in order to motivate them to keep engaging in it. Theories as to why people continue to engage in rumination despite its negative consequences vary. Some researchers argue that ruminative behavior is reinforced because it allows the ruminator to evade active engagement in problem-solving (Moulds, Kandris, Starr, \& Wong, 2007). Whatever the reason, the evidence suggests that some people do like to immerse themselves in negative emotions despite its negative consequences, even seeming to be "addicted" to such experiences (Garrido \& Schubert, 2011).

However, the data of the present study suggests that the relationship between rumination and enjoying being immersed in sadness is mediated by the individual's capacity for absorption. Absorption in itself is a healthy trait and an enjoyable experience (Agarwal \& Karabanna, 2000; Kreutz, Ott, Teichmann, Osawa, \& Vaitl, 2008; Rodriguez-Sanchez et al., 2011). Previous research indicates that people with high scores in absorption are able to enjoy the negative emotions aroused by sad music without experiencing the displeasure that would normally be associated with a sad event (Garrido \& Schubert, 2011). While absorption is normally considered an adaptive capacity, when accompanied by rumination (self-absorption), it may have the effect of exacerbating tendencies to become absorbed in one's own negative emotions, or in negative stimuli such as music. People with high scores in absorption who are also ruminators are likely the ones who tend to enjoy "wallowing" in negative emotions, whereas people with lesser capacities for absorption may be more likely to be aware of the negative consequences of listening to sad music.

These assertions are supported by the evidence found in this study that the enjoyment of listening to sad music (as measured by LSMS) and one's propensity to ruminate is mediated by absorption. The LSMS includes items that report motivations for listening to sad music that mostly relate to the believed benefits to be obtained from listening to it. For example, participants rate statements relating to enjoying the cathartic opportunity of listening to sad music, or using the opportunity to reflect on and process negative emotions being experienced. If these benefits are truly obtained, then a high score on the LSMS could indicate that music is being used in a healthy way to process grief or other negative emotions. However, if - as is likely in the case of a person who is ruminating using music - the believed benefits are not actually being obtained, then high LSMS scores could indicate that music is being used in maladaptive ways. In this study, ruminators who are also high in absorption demonstrated a liking for sad music. It seems likely that this effect is multiplied in ruminators who also have strong capacities for absorption. Such individuals may find it even more difficult to disengage from negative stimuli than others and may be less aware of the negative impact this is having on their mood and mental health, since the experience of absorption is in itself enjoyable. Therefore, it is possible that such individuals may find themselves strongly attracted to sad music despite its negative impact on them, and more likely to justify and rationalize their behavior.

Thus, for healthy listeners it is likely that the experience of sadness in music is not detrimental. Listening to music can be a positive coping skill for many people, especially people with a tendency to use music for reflective purposes who may use music as a tool for processing negative emotions being experienced (Garrido \& Schubert, 2013a). However, for people who also have a strong tendency to rumination and who have difficulty in disengaging from negative emotions, the ultimate effect upon mood may be detrimental. Possibly, the simultaneous existence of strong tendencies to absorption in an individual may cloud their judgment of the effect that the sad music is having on their mood.

This study is limited by the fact that it involved selfreport mood measures taken immediately after the listening stimuli. Conclusions cannot therefore be drawn about the effect of musical engagement upon long-term mood or clinical condition. However, it suggests, along with other prior research, that it cannot be assumed that the actual outcome of music listening is always as beneficial as claimed by participants. Further testing of this 
hypothesis should be conducted by measuring the effect upon mood over longer periods, perhaps even several weeks, targeting people with clinically assessed depression. Future studies would also benefit from using multiple methods for assessing mood in addition to self-report, such as physiological measures, behavioral analysis, and reports by caregivers. The study is further limited by the fact that participants were not questioned as to the extent to which their mood was impacted by the non-musical elements of the YouTube videos. In fact, it is not certain whether participants merely listened to the music, or also watched the accompanying video. Further studies would benefit from analyzing the level of engagement with visual elements of people who typically use YouTube or similar media delivery services as a source of music, and examining the relative impact of various kinds of videos on the mood-related outcome.

This paper has attempted to build theory to help better understand some of the nonadaptive uses of music. We reported a study that investigated how people feel, and what they believe they would feel when listening to sad music, and found that high ruminators are attracted to sad music. The attraction consistently led to a more depressed mood, even when some participants thought their mood would improve. Our theoretical proposal, which is supported by the data of this study, is that listeners are frequently attracted to sad music because they can enjoy the state of absorption they derive for such experiences. Further studies will be able to more closely investigate this idea. The current study intends to provoke debate on the neglected, theory-poor area of maladaptive uses of music.

The study therefore has implications for contemporary clinical practice in that it suggests that mental health professionals, including music therapists, should be educating patients about musical engagement as both a positive and potentially negative coping strategy. Further research could pursue this line of argument in looking at whether rumination or mental health state can also provide a possible explanation for the question of why certain genres of music such as rap or heavy metal sometimes have a detrimental effect on wellbeing and behavior, while at other times it appears to provide a useful coping method.

\section{Author Note}

Correspondence concerning this article should be addressed to Sandra Garrido, 151 Barry Street, University of Melbourne, Victoria 3010, Australia. E-mail: Sandra .garrido@unimelb.edu.au or E.Schubert@unsw.edu.au

\section{References}

Agarwal, R., \& Karabanna, E. (2000). Time flies when you're having fun: Cognitive absorption and beliefs about information technology usage 1. MIS Quarterly, 24, 665-694.

Barnhofer, T., Kuehn, E.-M., De Jong-Meyer, R., \& Williams, J. M. G. (2006). Beliefs about the benefits of rumination in depressed men and women with and without a history of assault. Behavioral and Cognitive Psychotherapy, 35, 317-324.

Beck, A. T., Baruch, E., \& Balter, D. M. (2004). A new instrument for measuring insight: The Beck Cognitive Insight Scale. Schizophrenia Research, 68, 319-329.

Beck, A. T., Ward, C. H., Mendelsohn, M., Mock, J., \& Erbaugh (1961). An inventory for measuring depression. Archives of General Psychiatry, 4, 561-571.

Bodner, E., Iancu, I., Gilboa, A., Sarel, A., Mazor, A., \& AMir, D. (2007). Finding words for emotions: The reactions of patients with major depressive disorder towards various musical excerpts. The Arts in Psychotherapy, 34, 142-150.

Calmes, C. A., \& Roberts, J. E. (2007). Repetitive thought and emotional distress: Rumination and worry as prospective predictors of depressive and anxious symptomatology. Cognitive Therapy and Research, 31, 343-356.
Chen, L., Zhou, S., \& Bryant, J. (2007). Temporal changes in mood repair through music consumption: Effects of mood, mood salience, and individual differences. Media Psychology, 9, 695-713.

Conway, M., Csank, P. A. R., Holm, S. L., \& Blake, C. K. (2000). On assessing individual differences in rumination on sadness. Journal of Personality Assessment, 75, 404-425.

Eerola, T., \& Vuoskoski, J. K. (2011). A comparison of the discrete and dimensional models of emotion in music. Psychology of Music, 39, 18-39.

EIsen, M. L., \& LynN, S. J. (2001). Dissociation, memory and suggestibility in adults and children. Applied Cognitive Psychology, 15, S49-S73.

Forbes, E. E., \& DAhl, R. C. (2005). Neural systems of positive affect: Relevance to understanding child and adolescent depression? Development and Psychopathology, 17, 827-850.

Garrido, S., \& Schubert, E. (2011). Negative emotion in music: What is the attraction? A qualitative study. Empirical Musicology Review, 6, 214-230.

Garrido, S., \& Schubert, E. (2013a). Adaptive and maladaptive attraction to negative emotion in music. Musicae Scientiae, 17, 145-164. 
Garrido, S., \& Schubert, E. (2013b). Moody melodies: Do they cheer us up? A study of the effect of sad music on mood. Psychology of Music, 43, 244-261.

Glisky, M. L., \& Kinlstrom, J. F. (1993). Hypnotizability and facets of openness. International Journal of Clinical and Experimental Hypnosis, 41, 112-123.

Gotlib, I. H., Krasnoperova, E., Neubauer Yue, D., \& Joormann, J. (2004). Attentional biases for negative interpersonal stimuli in clinical depression. Journal of Abnormal Psychology, 113, 127-135.

Joorman, J. (2005). Inhibition, rumination and mood regulation in depression. In R. W. Engle, G. Sedek, U. v. Hecker, \& D. N. McIntosh (Eds.), Cognitive limitations in aging and psychopathology: Attention, working memory, and executive functions (pp. 275-312). New York: Cambridge University Press.

Kreutz, G., Otт, U., Teichmann, D., Osawa, P., \& Vaitl, D. (2008). Using music to induce emotion: Influences of musical preference and absorption. Psychology of Music, 36, 101-121.

Lyubomirsky, S., Caldwell, N. D., \& Nolen-Hoeksema, S. (1998). Effects of ruminative and distractive responses to depressed mood on retrieval of autobiographical memories. Journal of Personality and Social Psychology, 75, 166-177.

McNair, D. M., Lorr, M., \& Droppleman, I. F. (1971). Manual for the profile of mood states. San Diego, CA: Educational and Industrial Testing Service.

Miranda, D., \& Claes, M. (2009). Music listening, coping, peer affiliation and depression in adolescence. Psychology of Music, 37, 215-233.

Moulds, M. L., Kandris, E., Starr, S., \& Wong, A. C. M. (2007). The relationship between rumination, avoidance and depression in a non-clinical sample. Behaviour Research and Therapy, 45, 251-261.

Nolen-Hoeksema, S. (1991). Responses to depression and their effects on the duration of depressive episodes. Journal of Abnormal Psychology, 100, 569-582.

Nolen-Hoeksema, S., \& Morrow, J. (1993). Effects of rumination and distraction on naturally occurring depressed mood. Cognition and Emotion, 7, 561-570.

Privette, G. (1983). Peak experience, peak performance nad flow: A comparative analysis of positive human experiences. Journal of Personality and Social Psychology, 45, 1361-1368.
Punkanen, M., Eerola, T., \& Erkkila, J. (2011). Biased emotional preferences in depression: Decreased liking of angry and energetic music by depressed patients. Music and Medicine, 3, 114-120.

Raes, F., Hermans, D., \& Williams, J. M. G. (2006). Negative bias in the perception of others' facial emotional expressions in major depression: The role of depressive rumination. Journal of Nervous and Mental Disease, 194, 796-799.

Riggs, S. E., Grant, P. M., Perivoloitis, D., \& Beck, A. T. (2012). Assessment of cognitive insight: A qualitative review. Schizophrenia Bulletin, 38, 338-350.

Rodriguez-Sanchez, A. M., Schauflei, W., Salanova, M., Cifre, E., \& Sonnenschein, M. (2011). Enjoyment and absorption: An electronic diary study on daily flow patterns. Work and Stress, 25, 75-92.

SaArikallio, S., \& Erkkila, J. (2007). The role of music in adolescents' mood regulation. Psychology of Music, 35, 88-109.

Thayer, R. E. (1978). Toward a psychological theory of multidimensional activation (arousal). Motivation and Emotion, 2, $1-34$.

Trapnell, P. D. (2010). BDI and RRQ rumination. Unpublished manuscript.

Trapnell, P. D., \& Campbell, J. D. (1999). Private selfconsciousness and the five-factor model of personality: Distinguishing rumination from reflection. Journal of Personality and Social Psychology, 76, 284-304.

VAN DEN Tol, A. J. M., \& Edwards, J. (2013). Exploring a rationale for choosing to listen to sad music when feeling sad. Psychology of Music, 41, 440-465.

Wenzlaff, R. M., \& Bates, D. E. (1998). Unmasking a cognitive vulnerability to depression: How lapses in mental control reveal depressive thinking. Journal of Personality and Social Psychology, 75, 1559-1571.

Wilhelm, K., Gillis, I., Schubert, E., \& Whittle, E. L. (2013). On a blue note: Depressed people's reasons for listening to music. Music and Medicine, 5, 76-83.

Wilkins, J. W., Hamby, S. L., Robertson, K. R., Knorr, K. L., Barry, N. S., \& Hall, C. D. (1995). The Profile of Mood States as a screening test for major depression in HIV+ patients. Assessment, 2, 181-188. 


\section{University Library}

\section{- M M I E E R VA A gateway to Melbourne's research publications}

Minerva Access is the Institutional Repository of The University of Melbourne

Author/s:

Garrido, S;Schubert, E

Title:

MUSIC AND PEOPLE WITH TENDENCIES TO DEPRESSION

Date:

2015-04-01

Citation:

Garrido, S. \& Schubert, E. (2015). MUSIC AND PEOPLE WITH TENDENCIES TO DEPRESSION. MUSIC PERCEPTION, 32 (4), pp.313-321. https://doi.org/10.1525/ MP.2015.32.4.313.

Persistent Link:

http://hdl.handle.net/11343/56456 